\title{
DevR (DosR) mimetic peptides impair transcriptional regulation and survival of Mycobacterium tuberculosis under hypoxia by inhibiting the autokinase activity of DevS sensor kinase
}

Kohinoor Kaur ${ }^{1+}$, Neetu Kumra Taneja ${ }^{1,2+}$, Sakshi Dhingra ${ }^{1}$ and Jaya S Tyagi ${ }^{1 *}$

\begin{abstract}
Background: Two-component systems have emerged as compelling targets for antibacterial drug design for a number of reasons including the distinct histidine phosphorylation property of their constituent sensor kinases. The DevR-DevS/DosT two component system of Mycobacterium tuberculosis (M. tb) is essential for survival under hypoxia, a stress associated with dormancy development in vivo. In the present study a combinatorial peptide phage display library was screened for DevS histidine kinase interacting peptides with the aim of isolating inhibitors of DevR-DevS signaling.
\end{abstract}

Results: DevS binding peptides were identified from a phage display library after three rounds of panning using DevS as bait. The peptides showed sequence similarity with conserved residues in the N-terminal domain of DevR and suggested that they may represent interacting surfaces between DevS and DevR. Two DevR mimetic peptides were found to specifically inhibit DevR-dependent transcriptional activity and restrict the hypoxic survival of M. tb. The mechanism of peptide action is majorly attributed to an inhibition of DevS autokinase activity.

Conclusions: These findings demonstrate that DevR mimetic peptides impede DevS activation and that intercepting DevS activation at an early step in the signaling cascade impairs $M$. tb survival in a hypoxia persistence model.

Keywords: Phage display, DevRS peptides, Inhibition of autokinase, Hypoxia

\section{Background}

The DevR dormancy regulon of Mycobacterium tuberculosis $(M . t b)$ is a transcriptional program induced by low oxygen tension, nitric oxide (NO), carbon monoxide (CO) or vitamin $\mathrm{C}$ treatment that enables bacterial adaptation and survival during periods of non-replicating persistence in in vitro models of dormancy [1-4]. This regulon is believed to assist bacterial survival during latent tuberculosis (TB) infection, a chronic asymptomatic state that afflicts one-third of the global population [5]. The expression of the $\sim 48$ gene regulon is coordinated by DevR (also known as DosR) which binds to target sites on DNA and induces transcription after its phosphorylation by DevS and/or

\footnotetext{
* Correspondence: jstyagi@gmail.com

${ }^{\dagger}$ Equal contributors

'Department of Biotechnology, All India Institute of Medical Sciences, New Delhi, India

Full list of author information is available at the end of the article
}

DosT [3,6-8]. DevS and DosT sensor kinases were therefore proposed as targets for the development of novel antibacterial compounds against dormant bacteria [9]. DevS was also predicted to be a potential persistence target inhibiting dormant $M$. tb using an in silico approach [10].

Two component systems are considered as compelling targets for drug design due to a number of reasons including their absence in higher eukaryotes, the difference in bacterial two-component signaling as compared to signaling pathways in eukaryotes, and most importantly, the essential roles they play in bacterial viability, virulence and drug resistance. Inhibitors of bacterial histidine kinases have been reported in the literature [11-13] but most suffer from the drawback of being extremely hydrophobic. Most inhibitors exhibit multiple mechanisms of action including surfactant and membrane damaging properties that are independent of two-component system inhibition. The 
high hydrophobicity of these molecules makes formulation and delivery of the compounds extremely difficult. Furthermore, the compounds showed excessive plasma protein binding and minimal bioavailability and were ineffective in standard in vivo infection models. In contrast, peptides are believed to confer several advantages, such as high target specificity, lower accumulation in tissues and lower toxicity coupled with new efficient synthesis strategies and low monomer prices. A recent study identified potential PhoQ inhibitor compounds that inhibited autophosphorylation and also inhibited severe keratoconjunctival inflammation in mice inoculated with Shigella flexneri [14], suggesting that two-component systems are potential targets for the development of drugs against bacteria.

We had earlier described the properties of a DevR binding peptide DevRS1 identified through phage display technology that inhibited DevR function [15]. Here we report the identification of peptides that majorly target the autokinase function of DevS sensor and inhibit DevR-mediated transcription and survival of $M$. $t b$ under hypoxia. The ability to interfere with DevR-DevS function at more than one step demonstrates that this twocomponent system is a rich target for developing inhibitor(s) to effectively block $M$. $t b$ adaptation to hypoxia, a potent dormancy signal.

\section{Results and discussion}

\section{Isolation of DevS binding peptides from a phage display} peptide library

The cytoplasmic domain of DevS (named as DevS 201 ), that transfers the activating phosphosignal to DevR [7], was used to screen a phage display peptide library using a panning strategy described in Figure 1. A total of 110 phages from the glycine eluate of $\mathrm{DevS}_{201}$ plate from the third round of panning were individually amplified and screened by ELISA to assess their DevS binding specificity. DevS binder phages were enriched relative to the unpanned library after three rounds of panning (Figure 2A). Thirty clones (G8, G9 etc.) showed 2-4 fold higher binding to $\mathrm{DevS}_{201}$ versus BSA or uncoated wells (Figure $2 \mathrm{~B}$ ). DNA sequence analysis of the peptide coding sequences in these phages revealed four peptide sequences, namely, HNTRGEE (Pep A), TFESYSL (Pep B), SLFRDWP (Pep C) and ITNPDPY (Pep D) that were repeated 2-4 times (Figure 2C).

\section{Activity of 'Pep' peptides}

The 'Pep' peptides identified by biopanning were tested for activity in DevS autokinase assays. Pep A, B and D peptides were found to inhibit DevS autokinase activity to varying degrees (see Additional file 1). However, the data showed a great variation and was not reproducible among the assay replicates for individual peptides. This

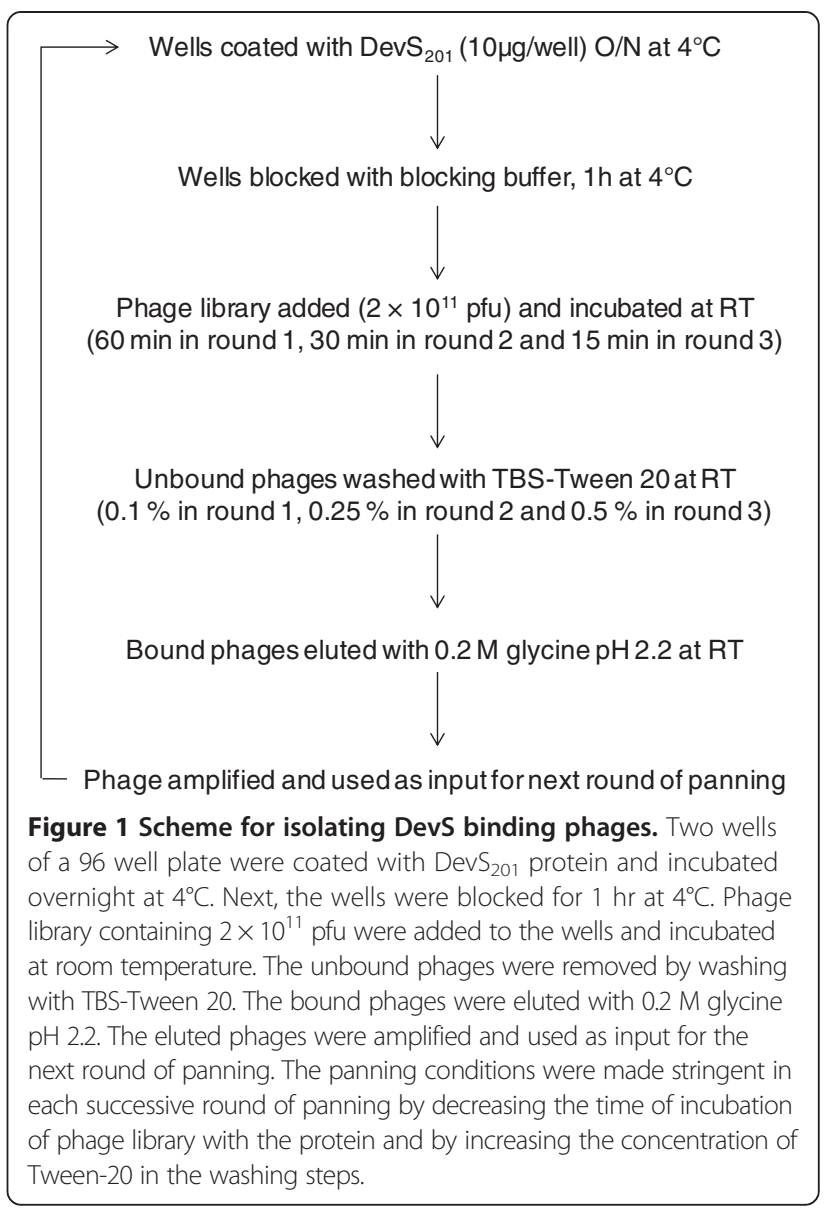

could be attributed to protein aggregation that was observed upon addition of peptides to the assays. However, Pep $\mathrm{C}$ did not inhibit autokinase activity at any of the concentrations tested.

\section{Similarity between PepA, PepB, PepC and PepD sequences and DevR catalytic centre}

The 'Pep' peptides identified by panning were aligned next with the protein sequence of DevR. Three peptides appeared to align near the Asp-54, Thr-82 and Lys-104 residues located in the $\mathrm{N}$-terminal domain of DevR (Figure 2C). Asp-54 is the site of phosphorylation and Thr-82 and Lys-104 residues are involved in phosphorylation-induced conformational changes $[7,16]$. The partial identity noted between 'Pep' peptides and DevR protein sequences suggested that these peptides might represent surfaces on the N-terminal domain of DevR $\left(\operatorname{DevR}_{N}\right)$ that could interact with DevS.

\section{The $\operatorname{DevR}_{\mathrm{N}}$ domain interacts with the cytosolic domain of DevS}

Because $\operatorname{DevR}_{\mathrm{N}}$, in particular its Asp54 residue, is involved in interaction of DevR and DevS proteins of $M$. smegmatis 

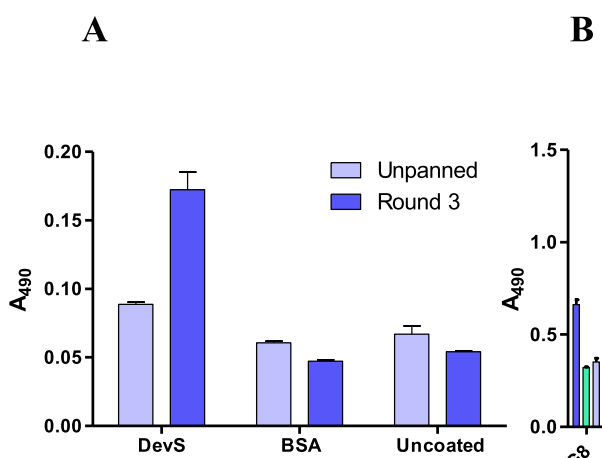

$\mathbf{C}$

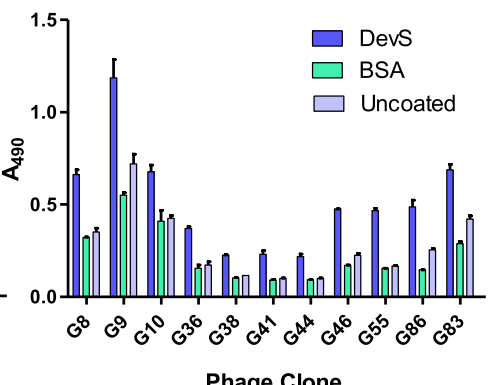

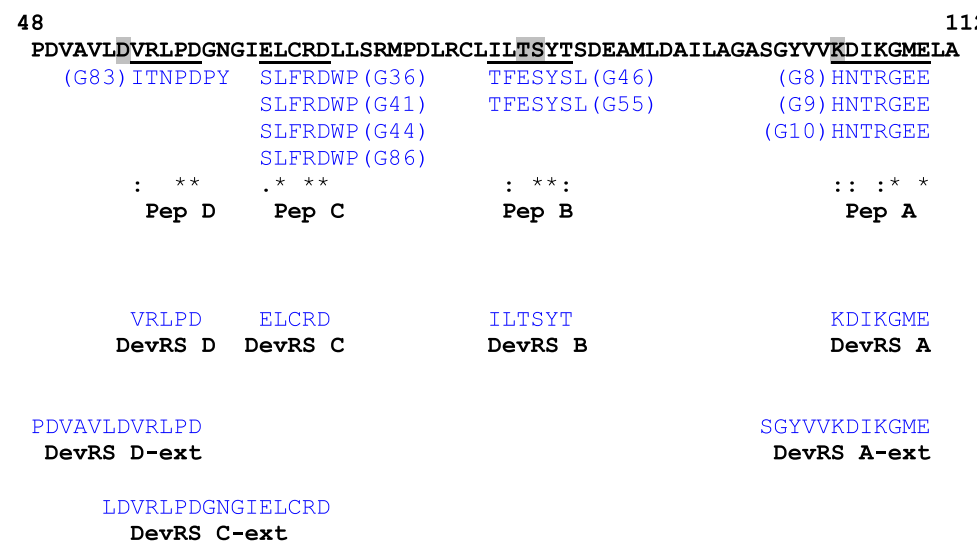

Figure 2 Identification of DevS binding peptides using biopanning. (A) Enrichment of phages binding to DevS protein. Equal numbers of phage particles $\left(10^{11}\right)$ before (unpanned library) and after three rounds of biopanning on DevS (Pan 3) were incubated in wells coated with DevS or BSA or left uncoated. The unbound phages were removed by washing the wells with TBS containing $0.5 \%$ Tween 20 and the bound phages were detected with HRP-conjugated anti-M13 antibody (Amersham) and o-Phenylenediamine substrate. The assays were performed in triplicate and the mean \pm SD values are plotted. (B) Phage ELISA of selected phage clones. Phage clones displaying DevS-binding peptides were used in ELISA. Equal numbers of phage particles after amplification were incubated in wells coated with DevS or BSA or left uncoated. The bound phages were detected as described above. (C) Alignment of peptides identified by DevS panning with the sequence of DevR. Functionally important and conserved residues: D54, phosphorylation site; T82, K104, residues involved in phosphorylation induced conformational changes, are highlighted in grey. Screening a phage display library for DevS binders identified the 'Pep' peptides. DevRS peptides were designed on the basis of DevR sequence. CLUSTALW-based alignment of the 'DevRS' peptides with the DevR sequence is shown. *, positions having a fully conserved residue; :, conservation between groups of strongly similar properties; •, conservation between groups of weakly similar properties. DevRS B-ext could not be analyzed due to the inability to prepare soluble peptide.

[17], we investigated the possibility of a similar interaction occurring between $\operatorname{DevR}_{\mathrm{N}}$ and DevS proteins of $M . t b$. An in vitro phosphotransfer assay was performed using $\operatorname{DevR}_{\mathrm{N}}$ and the cytosolic domain of DevS protein. A rapid and comparable transfer of the phosphosignal was noted from $M$. $t b$ DevS to either DevR or DevR $\mathrm{N}_{\mathrm{N}}$, which established that the $\mathrm{N}$ terminal domain of DevR interacts with DevS (Figure 3).

Having demonstrated that (1) 'Pep' peptides exhibit a modest anti-DevR activity (Additional file 1), (2) 'Pep' peptides bear sequence similarity with conserved sequences in the N-terminal domain of DevR (Figure 2C) and (3) $\operatorname{DevR}_{\mathrm{N}}$ interacts with DevS (Figure 3), we proceeded to synthesize 'DevR mimetic' peptides that mapped in the same regions as the 'Pep' peptides and were of identical sequence to DevR. The assumption was that DevR 'mimetic' peptides would interact with greater affinity with DevS as compared to the 'Pep' peptide sequences. The synthesized peptides were named as DevRS A (KDIKGME), DevRS B (ILTSYT), DevRS C (ELCRD) and DevRS D (VRLPD). Extended 10-16 mer versions of the DevRS peptides were also synthesized (peptides DevRS A-ext, C-ext, D-ext) assuming that they may afford better binding characteristics (Figure 2C). Due to the problem of protein aggregation observed in assays containing the 'Pep' series of peptides, only extended 'DevRS' and 'DevRS-ext' peptides were characterized further. Moreover, these extended peptides were likely 


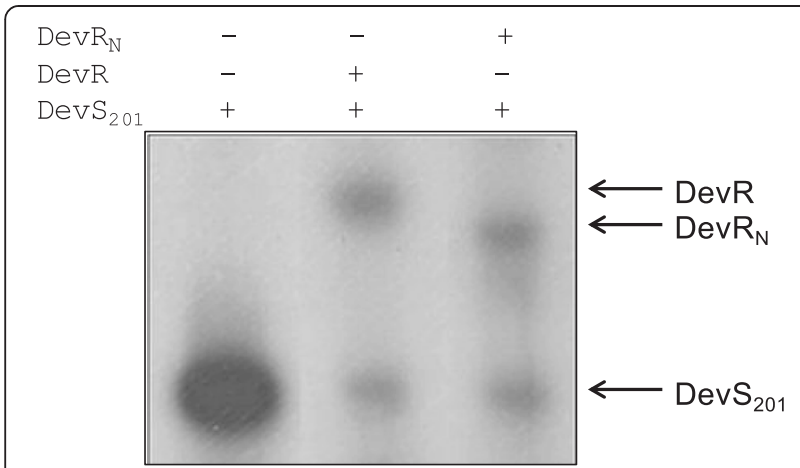

Figure $\mathbf{3}$ In vitro phosphotransfer assay. Reaction mixtures containing purified DevS $\sim{ }^{32} P$ and GST- DevR full length/GST - DevR $R_{N}$ proteins were incubated at $25^{\circ} \mathrm{C}$ for 2 mins. Samples were analysed by $15 \%$ SDS-PAGE and autoradiography.

to afford better binding characteristics if indeed they represented interacting regions.

\section{'DevRS' peptides inhibit DevR-mediated transcription}

DevRS A, B, C and D and DevRS A-ext, C-ext and D-ext peptides were assessed for their ability to inhibit DevR-regulated transcription using a $M$. $t b$ GFP reporter assay. The reporter assay measures the activity of the $R v 3134 c$ promoter, a well characterized DevRdependent promoter [18]. Two of the peptides that were tested, namely DevRS A-ext and DevRS D, significantly $(\mathrm{p}<0.05)$ inhibited $R v 3134 c$ promoter activity up to $\sim 40 \%$ under hypoxic conditions and $\sim 20-30 \%$ inhibition under aerobic conditions (Figure 4A and B).
Both these peptides map at or near the conserved sequences of DevR that are involved in phosphorylation and are believed to mediate a conformational change during its activation. The observed inhibition of promoter activity in aerobic cultures ( 20-30\%, Figure 4B) is ascribed to hypoxia development in standing cultures reported earlier and not to aerobic inhibition per se $[18,19]$. It was noteworthy that both DevRS A-ext and DevRS D peptides did not inhibit sigA promoter activity (not regulated by DevR) under identical conditions, establishing the target specificity of these peptides for DevR. However, the remaining peptides inhibited the $R v 3134 c$ promoter (up to $\sim 60 \%$ under hypoxia, Figure $4 \mathrm{~A}$ ), but caused non-specific inhibition of the sigA promoter and/or were inhibitory under aerobic conditions (up to $\sim 90 \%$ ), suggesting a lack of target specificity (Figure 4A and B). The specific inhibition of the Rv3134c promoter by DevRS A-ext and DevRS D peptides (Figure 4) implies that the expression of other members of the DevR regulon would also be impaired $[19,20]$ and accordingly these two peptides were characterized further in viability assays.

\section{'DevRS' peptides specifically inhibit hypoxic survival of $M$. tuberculosis}

DevRS signaling is established to be essential for $M$. $t b$ viability during hypoxia and not under aerobic conditions [21,22]. Therefore, DevRS A-ext and DevRS D peptides were evaluated for anti - TB activity using CFU assays and Resazurin reduction-based assays (REMA and HyRRA) as described in the Methods section. The REMA and HyRRA assays are very useful for screening anti-tubercular

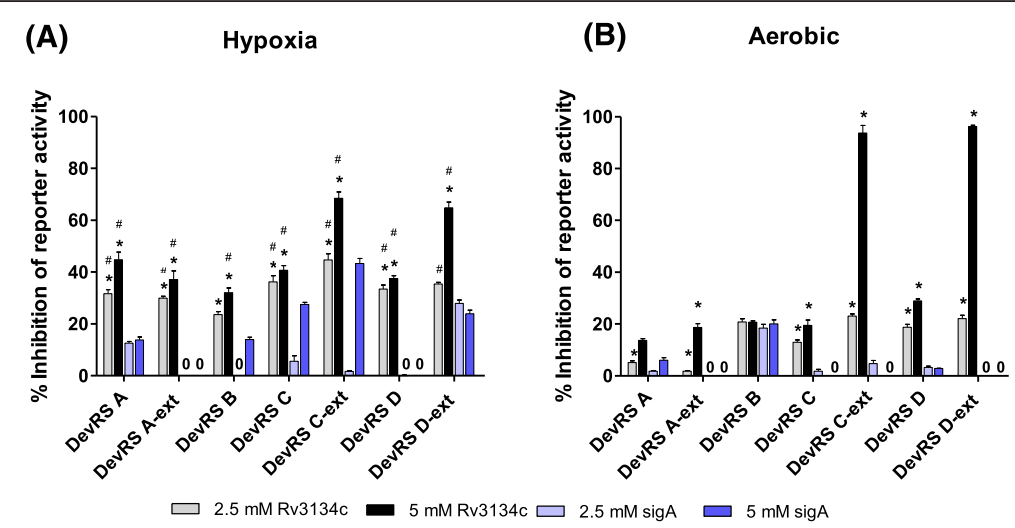

Figure 4 Peptides inhibit DevR-regulated promoter activity. Effect of peptides on Rv3134C and sigA promoter activity in M.tb cultures was assessed under hypoxic (A) and aerobic (B) conditions. The data represents the mean \pm SD of two independent experiments. * denotes a significant difference $(p<0.05)$ in the activity of Rv3134c and sigA promoters under hypoxic and aerobic conditions based on a Students' $t$-test. For example, DevRS A peptide-mediated inhibition (at $5 \mathrm{mM}$ concentration) of the Rv3134c promoter under hypoxic and aerobic conditions is $\sim 45 \%$ and $14 \%$, which is significantly higher than $~ 14 \%$ and $6 \%$ inhibition of sigA promoter activity under identical conditions. \# denotes a significant difference ( $p<0.05$ ) in Rv3134C promoter activity under hypoxic and aerobic conditions based on a Student's t-test. For example, DevRS A peptide-mediated inhibition (at $2.5 \mathrm{mM}$ and $5 \mathrm{mM}$ concentration) under hypoxia is 30\% and $45 \%$, which is significantly higher than 5\% \& 15\% inhibition observed under aerobic conditions. ' $\mathrm{O}$ ' values on top of some bars indicate no detectable inhibition. Non-specific inhibition of transcription (measured using sigA promoter reporter plasmid) was not observed for two peptides, DevRS A-ext and DevRS D peptides. 
anti-dormancy compounds under aerobic and hypoxic conditions respectively and can be safely performed in high-throughput format in M. smegmatis, $M$. bovis BCG and $M . t b$ [23]. In the CFU assay, DevRS A-ext and DevRS D peptides severely compromised hypoxic survival $M$. $t b$ ( $\sim 55$ to $95 \%$ inhibition with respect to the DMSO control) in contrast to only a modest reduction in CFU yields in aerobic cultures (none to $\sim 10 \%$ inhibition), thereby confirming target specificity (Figure 5A). The Resazurin reduction assays confirmed the target specificity of both the peptides; 40 to $70 \%$ loss in viability was observed in the presence of each of these peptides in the hypoxic HYRRA assay as compared to a modest inhibition in the aerobic REMA assay ( $15 \%$ to no inhibition, Figure $5 \mathrm{~B}$ and $\mathrm{C}$ ). These findings are consistent with our earlier observation that the HyRRA assay truly reports the viability of drug/inhibitor-treated dormant cultures [23]. A greater loss in peptide-mediated bacterial viability was observed in the CFU assay and it might be explained by the fact that inhibitor-treated bacteria were unable to grow on solid medium plates in the CFU assay but were maintained in viable form in broth cultures in the HyRRA/REMA assays as discussed earlier [23].

\section{'DevRS' peptides inhibit DevS autokinase activity}

Auto phosphorylation of the sensor kinase is the first step in the DevS/DosT-DevR signaling cascade. To decipher the mechanism of action of the peptides, DevRS A-ext and DevRS D were tested in an in vitro ATPdependent autokinase assay as described [7]. The addition of DevRS A-ext and DevRS D in the reaction inhibited DevS autokinase activity by $55 \%$ and $37 \%$ respectively (see Additional file 2). Our findings suggest that the inhibition of autokinase activity by DevRS A-ext and DevRS D is caused by peptide binding at/near the DevS phosphorylation site.
In addition to inhibiting autokinase activity (above), the peptides could in principle also inhibit transfer of the phosphosignal from DevS $\sim$ P to DevR. However, neither DevRS A-ext nor DevRS D inhibited this reaction in an in vitro phosphotransfer assay (data not shown). Based on these findings, the observed defects in hypoxic adaptation of $M . t b$ cultures are majorly attributed to a peptidemediated block in the autokinase activity of DevS sensor kinase. Because (1) of the high sequence homology between DevS and DosT kinases, (2) DosT, in addition to DevS, can activate DevR through its autokinase activity under hypoxia $[3,6,8,24]$ and (3) exposure to DevRS A-ext and DevRS D peptides results in a severe loss of hypoxic viability (Figure 5 ), we further propose that these two peptides inhibit the activities of both DevS and DosT kinases.

\section{Cytotoxicity of 'DevRS' peptides}

DevRS A-ext and DevRS D peptides were assessed next for their cytotoxicity in $\mathrm{CHO}$ and HepG2 mammalian cell lines using the MTT assay as described earlier [15]. Both the peptides were cytotoxic in the range of $20-40 \%$ at $5 \mathrm{mM}$ and $2.5 \mathrm{mM}$ concentration relative to the DMSO vehicle control (Figure 6).

\section{Conclusions}

In conclusion, the observed peptide-mediated inhibition, albeit at millimolar concentrations, demonstrates that interference of DevS/DosT signaling at the step of autokinase activity severely attenuates $M$. $t b$ adaptation and survival under hypoxia, a condition that prevails within granulomas and is a likely trigger for bacterial dormancy initiation and maintenance in vivo during latent TB. We have earlier demonstrated that phenylcoumarin- or peptide-mediated blocking of DevR function severely impairs $M$. $t b$ adaptation to hypoxia $[15,20]$. The small

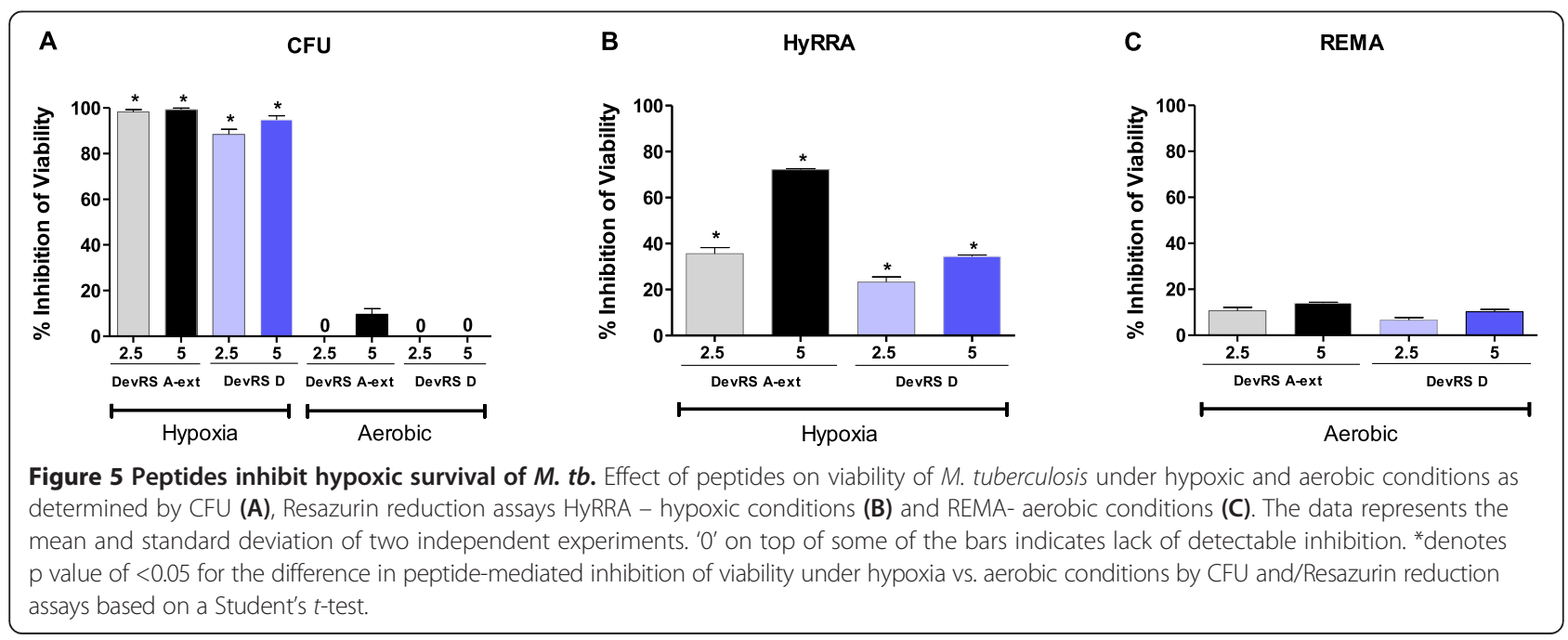




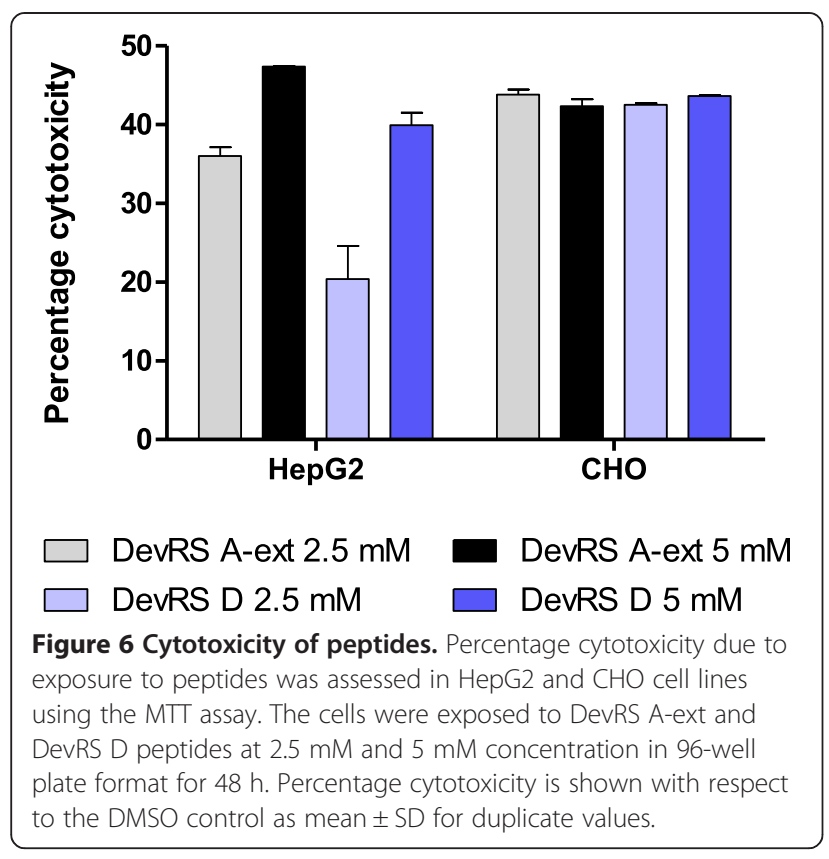

molecule inhibited DevR function by preventing its binding to specific motifs on DNA while the mode of action of the DevR binding peptide is yet unknown. In another approach, the isolated $\mathrm{N}$-terminal domain of DevR functioned as a novel competitive inhibitor that interfered with signal transfer from DevS to native DevR. Competitive signaling resulted in a defect in DevR mediated transcriptional response and a loss of hypoxic viability [21]. In the present study, we show that inhibition of DevS autokinase activity at an early step of the signaling cascade interferes with DevRS function and significantly reduces the hypoxic viability of bacteria. Thus the DevR-DevS system is a rich target to develop molecules that interfere with one of many steps in the signaling cascade during $M$. $t b$ adaptation to hypoxia (Figure 7). Our findings further suggest that phage display technology is a powerful approach for designing inhibitors and is likely to be useful for inhibiting the activity of other bacterial two-component systems.

\section{Methods}

\section{Screening of phage display library using cytoplasmic domain of DevS as bait}

The recombinant cytosolic portion of DevS protein $\mathrm{DevS}_{201}$ (amino acids 377 to 578), was overexpressed and purified from E. coli BL21 harboring recombinant plasmid pSCS201 as described earlier [7]. The Ph.D.-7 Phage display peptide library kit (New England Biolabs Inc., Beverely, MA, USA) was screened by biopanning using the manufacturer's instructions with a few modifications as outlined in Figure 1. Briefly, three rounds of panning were performed on a polystyrene 96-well micro titer plate wherein duplicate wells were coated with purified protein $\mathrm{DevS}_{201}-\mathrm{His}_{6}$ $(10 \mu \mathrm{g} / \mathrm{well})$ and the phage library $\left(2 \times 10^{11}\right.$ phages $)$ was incubated with the immobilized protein to allow binding of the phage particles. Decreasing the time of incubation with $\operatorname{DevS}_{201}$ protein and increasing the percentage of Tween-20 in the washing buffer in each successive round of panning achieved the stringent selection of high affinity binder phages. Three rounds of panning were performed in all and each time the phages in the glycine elution (0.2 M glycine, $\mathrm{pH} 2.2)$ were amplified and used as an input for the next round of panning. The titer of phages in the various elutions was determined according to the manufacturer's instructions and the input phage number was maintained during each round of panning.

\section{ELISA}

The high affinity binder phages were identified using ELISA. Briefly, individual phage plaques were picked from the glycine elution of the last round of panning, amplified and the culture supernatants (containing phages) were screened for binding to $\mathrm{DevS}_{201}$ or BSA or to plastic. Briefly, the plates were coated overnight with $10 \mu \mathrm{g} /$ well DevS $_{201}$ protein or left uncoated. The plates were then blocked overnight with BSA $(5 \mathrm{mg} / \mathrm{ml})$ followed by washing thrice with TBS $(50 \mathrm{mM}$ Tris- $\mathrm{HCl}, \mathrm{pH} 7.5$ and $150 \mathrm{mM}$ $\mathrm{NaCl}$ ). Thereafter, phage supernatants were added to coated wells and incubated for $1 \mathrm{hr}$. The plates were then washed vigorously with TBS containing 0.5\% Tween 20. The bound phages were detected using HRP-conjugated anti-M13 antibody (Amersham Biosciences, UK) and o-phenylenediamine as a substrate and measurement of absorbance at $\mathrm{A}_{490}$. The extent of binding to DevS was calculated as $\mathrm{A}_{490}$ in DevS coated wells $-\mathrm{A}_{490}$ in control wells.

\section{DNA sequencing of phages}

DNA of thirty high affinity binder phages was sequenced to determine the DevS binding peptide sequences. The desired peptides were then synthesized commercially with a purity level of $>98 \%$.

\section{Activity of 'DevRS' peptides in M. tb cultures}

M. $t b \mathrm{H} 37 \mathrm{Rv}$ was grown to logarithmic phase in Dubos broth containing $0.05 \%$ Tween 80 supplemented with $10 \%$ albumin dextrose complex (DTA medium) and sub-cultured with shaking at $220 \mathrm{rpm}$ to $\mathrm{A}_{595} \sim 0.5$. The culture was diluted in DTA medium (without Tween 80) to $A_{595} \sim 0.025$ for aerobic viability assays (REMA) and $\mathrm{A}_{595} \sim 0.005$ for hypoxic assays (HyRRA) and used as described earlier [15]. 

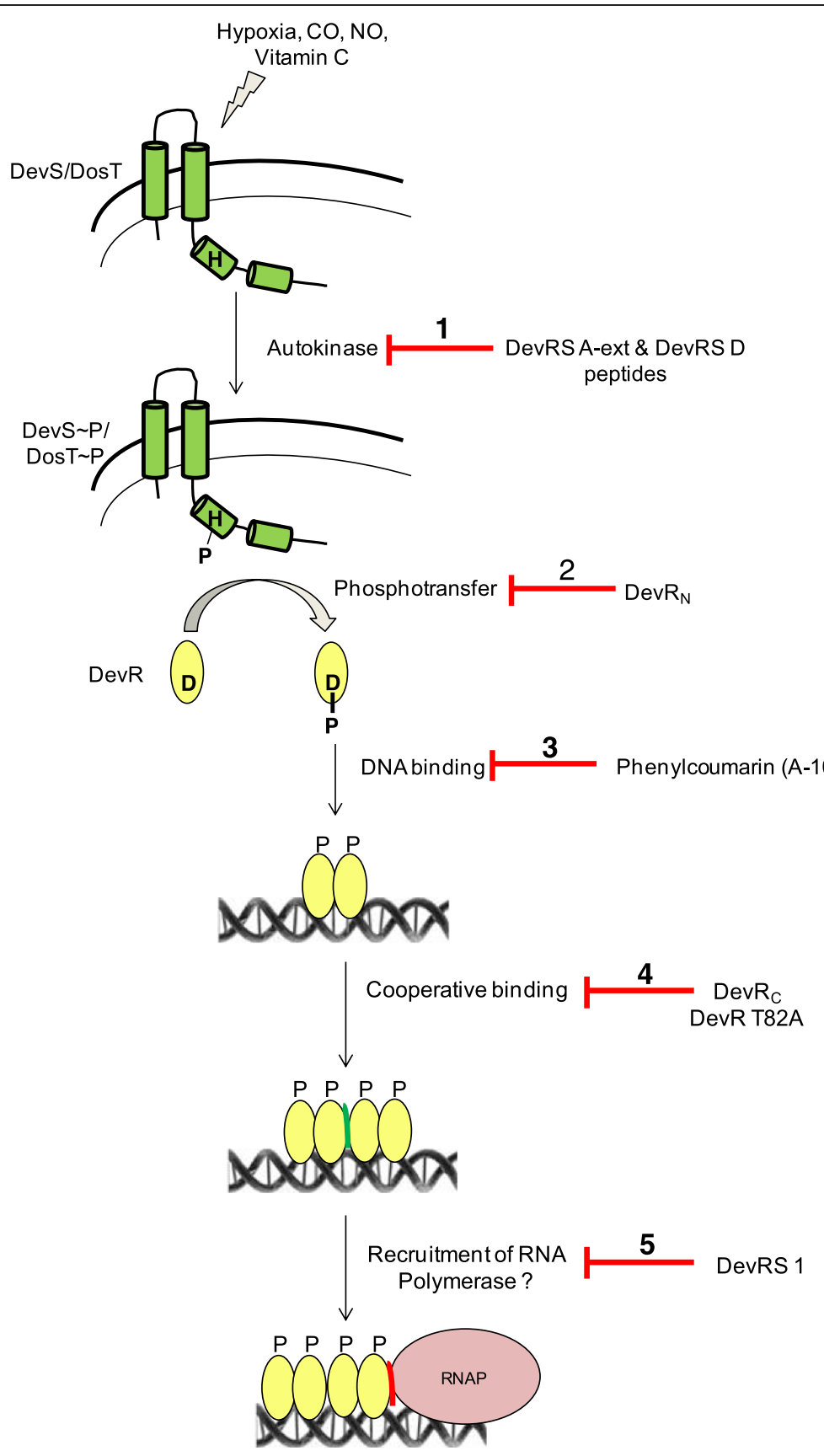

Figure 7 Interception of the DevRS signaling cascade. Hypoxia, NO, CO, and Vitamin C are sensed by DevS and DosT kinases (represented as membrane-associated sensors), resulting in the transfer of the phosphosignal from the sensors to DevR leading to its activation. Phosphorylated DevR binds to target gene promoters and elicits regulon activation. The red line/bars indicate the various steps in this signalling cascade whose interception results in a defect in the hypoxic adaptation of $M$. tb. Step 1, the inhibition of DevS/DosT autokinase activity by DevRS A-ext and DevRS D peptides (this study) interrupts the initial step in two-component signalling. Step 2, Dev $R_{N}$ protein competes with full-length DevR protein at the phosphotransfer step to inhibit bacterial adaptation [21]. Step 3, phenylcoumarin A-10 functions by inhibiting the binding of DevR to DNA [20]. Step 4, the cooperative interaction of DevR with DNA (green interface between 2 dimers of DevR) is required for robust induction [16,25], and serves as a novel inhibition step. Step 5, a DevR interacting peptide DevRS1 [15] likely exerts its inhibitory effect by blocking the between DevR - RNA polymerase interaction (red interface).

\section{REMA}

The REMA assay was performed in black, clear-bottomed, 96-well micro plates as described earlier [15]. Peptides were diluted in DMSO, and subsequent 2-fold serial dilutions were performed in $0.1 \mathrm{~mL}$ of Dubos medium supplemented with $0.05 \%$ glycerol in the micro plates. 
Approximately $5 \times 10^{4} \mathrm{cfu} M$. $t b$ bacteria were added per well. Appropriate control wells were included to calculate percentage inhibition of viability. The plates were incubated at $37^{\circ} \mathrm{C}$ for $64 \mathrm{~h}$ after which Resazurin $(0.003 \%$ final concentration) and Tween-80 (1.25\% final concentration) were added. The wells were observed after 24 and $48 \mathrm{~h}$ for a colour change from blue to pink and fluorescence of control wells $>50,000$ relative fluorescence units (RFU). Fluorescence was measured by excitation at $530 \mathrm{~nm}$ and emission at $590 \mathrm{~nm}$ using Gemini XS spectrofluorimeter in bottom reading mode. Percentage inhibition of viability was defined as: 1- (test well fluorescence/mean fluorescence of triplicate wells containing only bacteria) $\times 100$.

\section{HyRRA}

The HyRRA assay was performed as described earlier [15]. Briefly, $3 \mathrm{~mL}$ culture aliquots $\left(\mathrm{A}_{595} \sim 0.003\right.$ containing $\left.\sim 1.5 \times 10^{6} \mathrm{cfu} / \mathrm{mL} M . t b \mathrm{H} 37 \mathrm{Rv}\right)$ were injected into $5 \mathrm{~mL}$ uncoated Vacutainer tubes and kept stationary at $37^{\circ} \mathrm{C}$ to allow for self-generation of hypoxia in the cultures. The peptides were injected into anoxic cultures (as judged by decolorization of methylene blue) at different concentrations on day 30 and the tubes were further incubated for 5 days at $37^{\circ} \mathrm{C}$. Resazurin (0.0023\% final concentration) and Tween 80 (0.66\%/well final concentration) were injected into each tube and the tubes were incubated overnight. Culture aliquots $(200 \mu \mathrm{l})$ were transferred to a 96-well black micro plate and fluorescence determined as described above.

\section{GFP Reporter assays}

For the reporter assays, $200 \mu \mathrm{l} M$. $t b$ culture aliquots harboring p3134c-1/pSigA reporter plasmids were used to measure GFP fluorescence in a spectrofluorimeter in bottom reading mode using an excitation wavelength of $483 \mathrm{~nm}$ and an emission wavelength of $515 \mathrm{~nm}$ as described [18].

\section{Auto phosphorylation of DevS 201}

$\operatorname{DevS}_{201}(15 \mu \mathrm{M}$ final concentration) was incubated with $5 \mu \mathrm{Ci}$ of $\gamma^{32} \mathrm{P}$ ATP $\left(5000 \mathrm{Ci} \mathrm{mmol}^{-1}\right.$, BRIT, India) in a 10 $\mu \mathrm{l}$ reaction mixture (containing $50 \mu \mathrm{M}$ Tris $\mathrm{HCl} \mathrm{pH} \mathrm{8.0,}$ $50 \mathrm{mM} \mathrm{KCl}, 10 \mathrm{mM} \mathrm{MgCl}_{2}, 50 \mu \mathrm{M} \mathrm{ATP}$ ) at $25^{\circ} \mathrm{C}$ for 60 mins. The reactions were stopped by dilution with 200 $\mu \mathrm{l}$ of cold phosphate-buffered saline (PBS). The diluted contents were promptly transferred to a 96-well MultiScreen HA-plate prewetted with PBS and processed by high-throughput filtration using a vacuum manifold device to remove the reaction components and trap the proteins on the membrane [9]. The wells were washed thrice with $300 \mu \mathrm{l}$ PBS using the vacuum manifold. The filters were subsequently dried on the vacuum for 2 mins at room temperature and individual filters were cut using the filter removal device. The radioactivity that was retained on each filter was quantitated using liquid scintillation counting. To monitor the effect of peptides on $\mathrm{DevS}_{201}$ autokinase activity, the peptides at various concentrations were preincubated with $\mathrm{DevS}_{201}$ in the reaction mixture for 30 mins at $25^{\circ} \mathrm{C}$. ATP was then added to this reaction cocktail and tubes were incubated at $25^{\circ} \mathrm{C}$ for $1 \mathrm{~h}$. The reaction was terminated by dilution with $200 \mu \mathrm{l}$ of cold PBS and the samples were analyzed as described above.

\section{Phosphorylation of DevR/DevR $R_{N}$ by phosphotransfer from DevS 201}

To study the phosphotransfer reaction, $\operatorname{DevS}_{201}(15 \mu \mathrm{M}$ final concentration) was phosphorylated for 60 mins as described above. Subsequently, full-length DevR or DevR ${ }_{N}$ protein (1-144 amino acid residues, $20 \mu \mathrm{M}$ final concentration) was added to the phosphorylated $\mathrm{DevS}_{201} \mathrm{mix}$, and the reaction was incubated at $25^{\circ} \mathrm{C}$ for 2 mins. The reaction was terminated with $10 \mu \mathrm{l}$ of $2 \mathrm{X}$ stop buffer (100 mM Tris-Cl, pH 6.8, 20\% glycerol, 2\% SDS, 280 $\mathrm{mM} \beta$-mercaptoethanol, 0.01\% bromophenol blue) and the samples were resolved on SDS-PAGE. The gel was rinsed in water and subjected to autoradiography.

\section{Cytotoxicity assay}

The cytotoxicity of DevRS1 peptide was assessed in CHO (Chinese hamster ovary cells) and HepG2 (human liver hepatocellular carcinoma) cell lines. Both the cell lines were maintained in DMEM supplemented with $10 \% \mathrm{FBS}$ at $37^{\circ} \mathrm{C}$ in $5 \% \mathrm{CO}_{2}$. Approximately, $10^{4}$ cells were seeded per well in a 96-well plate and incubated at $37{ }^{\circ} \mathrm{C}$ for $12-16 \mathrm{~h}$. The peptides were diluted in $125 \mu \mathrm{l}$ DMEM and added onto cells (final volume $250 \mu \mathrm{l}$ per well, 2.5 and $5 \mathrm{mM}$ final peptide concentrations), and the plate was incubated at $37^{\circ} \mathrm{C}$ in $5 \% \mathrm{CO}_{2}$ for $48 \mathrm{~h}$. Subsequently, $20 \mu \mathrm{l}$ of MTT (Sigma $5 \mu \mathrm{g} \mathrm{ll}^{-1}$ ) was added and incubated for $4-5 \mathrm{~h}$ at $37^{\circ} \mathrm{C}$. Following incubation, media was discarded and the formazan crystals were solubilized by adding $200 \mu \mathrm{l}$ DMSO and the absorbance measured at $\mathrm{A}_{560} \mathrm{~nm}$. The percentage toxicity was calculated as:

$$
1-\frac{A_{\text {peptide }}}{A_{\text {DMSO }}} \times 100
$$

\section{Additional files}

Additional file 1: 'Pep' peptides inhibit DevS autokinase activity. To assess the effect of Pep A, B, C and D peptides on autokinase activity of DevS $_{201}$, the peptides were incubated with DevS $S_{201}$ protein at $3 \mathrm{mM}$ and $5 \mathrm{mM}$ concentrations in an autokinase reaction. The reactions were analyzed in a high throughput format as described in Methods. The data represents the mean \pm SD of three independent experiments. The number 0 on top of some of the bars indicates the lack of inhibition. The reproducibility was poor due to protein aggregation on addition of peptides to the autokinase reactions. 
Additional file 2: Effect of DevRS A-ext and DevRS D on autokinase activity of DevS $S_{201}$. The peptides were incubated with DevS 201 protein at $5 \mathrm{mM}$ concentration in an autokinase reaction and the reactions were then analyzed in a high throughput format as described in Methods. The data represents the mean \pm SD of two independent experiments.

\section{Competing interests}

The authors declare that they have no competing interests.

\section{Authors' contribution}

KK performed the biopanning procedure, phage screening and autokinase assays and drafted the manuscript. NKT carried out the reporter assays and REMA and HyRRA assays. SD participated in phage screening and performed the cytotoxicity experiments. JST contributed to the overall study design and manuscript preparation. All authors read and approved the final manuscript.

\section{Acknowledgements}

This study was financially supported by a grant to J.S.T. from CSIR, Government of India. We acknowledge the valuable suggestions of $\mathrm{Dr}$ Deepak Saini and Dr Deepti Saini during phage library screening. We thank Dr. Santosh Chauhan for providing DevR $R_{N}$ protein. J.S.T. is thankful to the DST for the J.C. Bose National Fellowship. K.K., N.K.T. and S.D. are thankful to UGC, CSIR and DBT respectively, for their Research Fellowships.

\section{Author details}

'Department of Biotechnology, All India Institute of Medical Sciences, New Delhi, India. ${ }^{2}$ Present address: National Institute of Food Technology Entrepreneurship and Management (Ministry of Food Processing Industries, Government of India) Plot No. 97, Sector-56, HSIIDC Industrial Estate, Kundli, District Sonepat, Sonepat, Haryana 131028, India.

Received: 28 March 2014 Accepted: 11 July 2014

Published: 21 July 2014

\section{References}

1. Kumar A, Deshane JS, Crossman DK, Bolisetty S, Yan BS, Kramnik I, Agarwal A, Steyn AJ: Heme oxygenase-1-derived carbon monoxide induces the Mycobacterium tuberculosis dormancy regulon. J Biol Chem 2008 283(26):18032-18039.

2. Sherman DR, Voskuil M, Schnappinger D, Liao R, Harrell MI, Schoolnik GK Regulation of the Mycobacterium tuberculosis hypoxic response gene encoding alpha -crystallin. Proc Natl Acad Sci U S A 2001, 98(13):7534-7539.

3. Taneja NK, Dhingra S, Mittal A, Naresh M, Tyagi JS: Mycobacterium tuberculosis transcriptional adaptation, growth arrest and dormancy phenotype development is triggered by vitamin C. PLoS One 2010 5(5):e10860.

4. Voskuil MI, Schnappinger D, Visconti KC, Harrell MI, Dolganov GM, Sherman DR, Schoolnik GK: Inhibition of respiration by nitric oxide induces a Mycobacterium tuberculosis dormancy program. J Exp Med 2003, 198(5):705-713.

5. Bretl DJ, Demetriadou C, Zahrt TC: Adaptation to environmental stimuli within the host: two-component signal transduction systems of Mycobacterium tuberculosis. Microbiol Mol Biol Rev 2011, 75(4):566-582.

6. Roberts DM, Liao RP, Wisedchaisri G, Hol WG, Sherman DR: Two sensor kinases contribute to the hypoxic response of Mycobacterium tuberculosis. J Biol Chem 2004, 279(22):23082-23087.

7. Saini DK, Malhotra V, Dey D, Pant N, Das TK, Tyagi JS: DevR-DevS is a bona fide two-component system of Mycobacterium tuberculosis that is hypoxia-responsive in the absence of the DNA-binding domain of DevR. Microbiology 2004, 150(Pt 4):865-875.

8. Saini DK, Malhotra $V$, Tyagi JS: Cross talk between DevS sensor kinase homologue, Rv2027c, and DevR response regulator of Mycobacterium tuberculosis. FEBS Lett 2004, 565(1-3):75-80.

9. Saini DK, Tyagi JS: High-throughput microplate phosphorylation assays based on DevR-DevS/Rv2027c 2-component signal transduction pathway to screen for novel antitubercular compounds. J Biomol Screen 2005, 10(3):215-224

10. Hasan S, Daugelat S, Rao PS, Schreiber M: Prioritizing genomic drug targets in pathogens: application to Mycobacterium tuberculosis. PLoS Comput Biol 2006, 2(6):e61.
11. Macielag MJ, Goldschmidt R: Inhibitors of bacterial two-component signalling systems. Expert Opin Investig Drugs 2000, 9(10):2351-2369.

12. Matsushita M, Janda KD: Histidine kinases as targets for new antimicrobial agents. Bioorg Med Chem 2002, 10(4):855-867.

13. Stephenson K, Hoch JA: Developing inhibitors to selectively target two-component and phosphorelay signal transduction systems of pathogenic microorganisms. Curr Med Chem 2004, 11(6):765-773.

14. Cai X, Zhang J, Chen M, Wu Y, Wang X, Chen J, Shen X, Qu D, Jiang H: The effect of the potential PhoQ histidine kinase inhibitors on Shigella flexneri virulence. PLoS One 2012, 6(8):e23100.

15. Dhingra S, Kaur K, Taneja NK, Tyagi JS: DevR (DosR) binding peptide inhibits adaptation of Mycobacterium tuberculosis under hypoxia. FEMS Microbiol Lett 2012, 330(1):66-71.

16. Gautam US, Sikri K, Tyagi JS: The residue threonine 82 of DevR (DosR) is essential for DevR activation and function in Mycobacterium tuberculosis despite its atypical location. J Bacterio/ 2011, 193(18):4849-4858.

17. Lee HN, Lee NO, Ko IJ, Kim SW, Kang BS, Oh JI: Involvement of the catalytically important Asp54 residue of Mycobacterium smegmatis DevR in protein-protein interactions between DevR and DevS. FEMS Microbiol Lett 2013, 343(1):26-33.

18. Chauhan S, Tyagi JS: Cooperative binding of phosphorylated DevR to upstream sites is necessary and sufficient for activation of the Rv3134c-devRS operon in Mycobacterium tuberculosis: implication in the induction of DevR target genes. J Bacteriol 2008, 190(12):4301-4312.

19. Chauhan S, Sharma D, Singh A, Surolia A, Tyagi JS: Comprehensive insights into Mycobacterium tuberculosis DevR (DosR) regulon activation switch. Nucleic Acids Res 2011, 39(17):7400-7414

20. Gupta RK, Thakur TS, Desiraju GR, Tyagi JS: Structure-based design of DevR inhibitor active against nonreplicating Mycobacterium tuberculosis. J Med Chem 2009, 52(20):6324-6334.

21. Majumdar SD, Sharma D, Vashist A, Kaur K, Taneja NK, Chauhan S, Challu VK, Ramanathan VD, Balasangameshwara V, Kumar P, Tyagi JS: Co-expression of DevR and $\operatorname{DevR}(\mathrm{N})$-Aph proteins is associated with hypoxic adaptation defect and virulence attenuation of Mycobacterium tuberculosis. PLOS One 2010, 5(2):e9448.

22. Majumdar SD, Vashist A, Dhingra S, Gupta R, Singh A, Challu VK, Ramanathan VD, Kumar P, Tyagi JS: Appropriate DevR (DosR)-mediated signaling determines transcriptional response, hypoxic viability and virulence of Mycobacterium tuberculosis. PLoS One 2012, 7(4):e35847.

23. Taneja NK, Tyagi JS: Resazurin reduction assays for screening of anti-tubercular compounds against dormant and actively growing Mycobacterium tuberculosis, Mycobacterium bovis BCG and Mycobacterium smegmatis. J Antimicrob Chemother 2007, 60(2):288-293.

24. Honaker RW, Leistikow RL, Bartek IL, Voskuil MI: Unique roles of DosT and DosS in DosR regulon induction and Mycobacterium tuberculosis dormancy. Infect Immun 2009, 77(8):3258-3263.

25. Gautam US, Chauhan S, Tyagi JS: Determinants outside the DevR C-termina domain are essential for cooperativity and robust activation of dormancy genes in Mycobacterium tuberculosis. PLoS One 2011, 6(1):e16500.

\section{doi:10.1186/1471-2180-14-195}

Cite this article as: Kaur et al:: DevR (DosR) mimetic peptides impair transcriptional regulation and survival of Mycobacterium tuberculosis under hypoxia by inhibiting the autokinase activity of DevS sensor kinase. BMC Microbiology 2014 14:195.

\section{Submit your next manuscript to BioMed Central and take full advantage of:}

- Convenient online submission

- Thorough peer review

- No space constraints or color figure charges

- Immediate publication on acceptance

- Inclusion in PubMed, CAS, Scopus and Google Scholar

- Research which is freely available for redistribution 